\title{
A polyphenol extract modifies quantity but not quality of liver fatty acid content in high-fat-high-sucrose diet-fed rats: possible implication of the sirtuin pathway
}

\author{
Manar Aoun ${ }^{1,2}$, Francoise Michel $^{2}$, Gilles Fouret ${ }^{1}$, Francois Casas ${ }^{1}$, Melanie Jullien ${ }^{1}$, \\ Chantal Wrutniak-Cabello ${ }^{1}$, Jeanne Ramos ${ }^{3}$, Jean-Paul Cristol ${ }^{2}$, Charles Coudray ${ }^{1}$, \\ Marie-Annette Carbonneau ${ }^{2}$ and Christine Feillet-Coudray ${ }^{1 *}$ \\ ${ }^{1}$ INRA UMR 866, Différentiation Cellulaire et Croissance, 34060 Montpellier, France \\ ${ }^{2}$ UMR 204 NUTRIPASS IRD/Montpellier-1/Montpellier-2/Supagro, 34000 Montpellier, France \\ ${ }^{3}$ Laboratoire d'Anatomie Pathologique, CHU Gui de Chauliac, 80, Avenue Augustin Fliche, 34295 Montpellier Cedex 5, France
}

(Received 15 December 2009 - Revised 1 June 2010 - Accepted 7 June 2010 - First published online 2 August 2010)

High-fat or high-fat-high-sucrose diets are known to induce non-alcoholic fatty liver disease and this is emerging as one of the most common liver diseases worldwide. Some polyphenols have been reported to decrease rat hepatic lipid accumulation, in particular those extracted from red grapes such as resveratrol. The present study was designed to determine whether a polyphenol extract (PPE), from red grapes, modulates liver fatty acid composition and desaturase activity indexes in rats fed a high-fat-high-sucrose (HFHS) diet, and to explore whether sirtuin-1 deacetylase activation was implicated in the effect of the PPE against liver steatosis. The effect of this PPE on mitochondriogenesis and mitochondrial activity was also explored. The PPE decreased liver TAG content in HFHS + PPE diet-fed rats in comparison with HFHS diet-fed rats. The PPE had no effect on liver fatty acid composition, desaturase activity indexes and stearoyl-CoA desaturase 1 (SCD1) gene expression. Sirtuin-1 deacetylase protein expression was significantly increased with the PPE; AMP kinase protein expression was higher with the PPE in comparison with the HFHS rats, but no modification of phosphorylated AMP kinase was observed. Protein expression of phospho-acetyl-CoA carboxylase was decreased in HFHS rats and returned to basal values with the PPE. Finally, the PPE modulated PPAR $\gamma$ coactivator-1 $\alpha$ (PGC-1 $\alpha$ ) but did not modify mitochondriogenesis and mitochondrial activity. In conclusion, the PPE partially prevented the accumulation of TAG in the liver by regulating acetyl-CoA carboxylase phosphorylation, a key enzyme in lipid metabolism, probably via sirtuin-1 deacetylase activation. However, the PPE had no effect on the qualitative composition of liver fatty acids.

Polyphenols: High-fat-high-sucrose diet: Sirtuin-1: Mitochondria: Oxidative stress: Fatty acids

The incidence of obesity and insulin resistance is increasing rapidly in wealthy societies, and has become a major public health problem, essentially resulting from imbalanced energy intake and expenditure of the body from a metabolic point of view ${ }^{(1)}$. This trend is associated with a parallel increase in the prevalence of non-alcoholic fatty liver disease

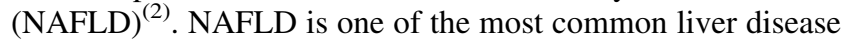
disorders, having a spectrum ranging from simple steatosis to cirrhosis $^{(3)}$. The leading hypothesis for NAFLD is the two-hit hypothesis. The 'first' hit is the accumulation of lipids in the hepatocytes (steatosis) and the 'second' hit leads to hepatocyte injury, inflammation and fibrosis (steatohepatitis/cirrhosis). Some of the probable second hits include oxidative stress, mitochondrial dysfunction and insulin resistance ${ }^{(4,5)}$.

Recent studies demonstrated that some polyphenols ${ }^{(6-10)}$ or polyphenol extracts (PPE) ${ }^{(11)}$ may prevent, at least partially, hepatic steatosis induced by high-fat-high-sugar Western diet administration. Indeed, polyphenols may act on lipid metabolism by modulating desaturase activity. For example, some polyphenols have been shown to reduce the gene expression of $\Delta-9$ desaturase (stearoyl-CoA desaturase 1; $S C D 1)^{(12,13)}$ and to decrease the activity of $\Delta-6$ desaturase $^{(14)}$. Nevertheless, whether polyphenols may modulate not only liver total lipid content but also liver fatty acid composition has never been addressed.

Polyphenols may also act on lipid metabolism signalling pathways, including lipid synthesis and degradation. Different reports $^{(15,16)}$ showed that elevated glucose inhibited AMP kinase activity and acetyl-CoA carboxylase phosphorylation and thus increased hepatocellular lipid accumulation. However, supplementation with polyphenols reversed the inhibition of AMP-activated protein kinase activity and acetyl-CoA carboxylase phosphorylation ${ }^{(15)}$. Moreover, they demonstrated that polyphenols stimulated AMP kinase and

Abbreviations: HFHS, high-fat-high-sucrose; HFHS + PPE, high-fat-high-sucrose and polyphenol extract; NAFLD, non-alcoholic fatty liver disease;

PGC-1 $\alpha$, PPAR $\gamma$ coactivator-1 $\alpha$; PPE, polyphenol extract; SCD1, stearoyl-CoA desaturase 1; SREBP-1c, sterol regulatory element-binding protein-1c.

* Corresponding author: Dr Christine Feillet-Coudray, fax +334675456 94, email cfeillet@supagro.inra.fr 
prevented hepatocyte lipid accumulation probably by activating sirtuin-1 deacetylase ${ }^{(16)}$.

As mitochondria are both a major site for fat metabolism and the main source of reactive oxygen and/or nitrogen species in hepatocytes, they are postulated to play a central role in the progression of NAFLD to non-alcoholic steatohepatitis and cirrhosis ${ }^{(5)}$. Some polyphenols have also been shown to modify mitochondrial activity and increase mitochondriogenesis $^{(17)}$. Such an effect may enhance mitochondrial lipid catabolism and hence attenuate liver fatty acid accumulation.

The present study was thus designed to determine whether a PPE modulates liver fatty acid content and their composition and desaturase activity indexes in rats fed a high-fat-highsucrose diet, and to explore whether the sirtuin-1-AMP kinase signalling pathway is implicated in the preventive effect of this PPE against liver steatosis. Moreover, as mitochondrial dysfunction may be implicated in the progression of steatosis to non-alcoholic steatohepatitis, the effect of the PPE on mitochondriogenesis and mitochondrial activity was also explored.

\section{Materials and methods}

\section{Animals and diets}

All animal experiments were performed according to European directives (86/609/CEE) and approved by the Comité d'Ethique en Matière d'Expérimentation Animale: Région Languedoc-Roussillon. Male Wistar rats ( $n$ 18; Charles River, L'Arbresle, France), aged 6 weeks, were used in the present study. Rats were housed two animals per cage under conditions of constant temperature $\left(20-22^{\circ} \mathrm{C}\right)$, humidity $(45-50 \%)$ and a standard dark cycle (from 20.00 until 08.00 hours). Our institution guidelines for the care and use of laboratory animals were observed. The rats were randomised into three groups of six animals: (1) a control group was fed for 6 weeks a control semi-purified diet; (2) a high-fat-high-sucrose (HFHS) group was fed for 6 weeks a high-fat-high-sucrose diet; (3) a high-fat-high-sucrose diet plus PPE (HFHS + PPE) group was fed for 6 weeks a highfat-high-sucrose diet containing $2 \mathrm{~g}$ PPE (Provinol $^{\mathrm{TM}}$; Société Française de Distillerie, Vallon Pont d'Arc, France) per kg diet. The period of 6 weeks was chosen because it was described as sufficient to induce hepatic steatosis in rats ${ }^{(18,19)}$. The control diet contained the following $(\mathrm{g} / \mathrm{kg})$ : casein, 200; starch, 660.7; soyabean oil, 40; cellulose, 50; mineral mix, $35^{(20)}$; vitamin mix, $10^{(20)}$; L-cystine, 1.8 ; choline bitartrate, 2.5. The HFHS diet contained the following $(\mathrm{g} / \mathrm{kg})$ : casein, 200; starch, 100.7; sucrose, 300; soyabean oil, 40; olive oil, 100; coprah oil, 150; cellulose, 50; mineral mix, $35^{(20)}$; vitamin mix, $10^{(20)}$; L-cystine, 1.8 ; choline bitartrate, 2.5. The HFHS + PPE diet was the same as the HFHS diet, plus $2 \mathrm{~g}$ PPE $/ \mathrm{kg}$ diet. The detailed fatty acid composition of the diets is given in Table 1. Rats were given free access to distilled water, and food, body growth and diet consumption were determined weekly. Food intake was determined as follows: dry rejected food was subtracted from dry presented food. The dry material percentage in the presented food was determined by drying a small amount of each presented diet $\left(3 \mathrm{~d}\right.$ at $\left.80^{\circ} \mathrm{C}\right)$ and weighing before and after drying. The presented food was then determined by multiplying the weight of the presented fresh food by the dry material percentage. The rejected food was dried $\left(3 \mathrm{~d}\right.$ at $\left.80^{\circ} \mathrm{C}\right)$ and weighed. The consumed dry material was calculated as follows: presented dry food - rejected dry food. The result was divided by 2 to take into account the presence of two rats per cage.

Provinol $^{\mathrm{TM}}$ is a powdered PPE obtained from red wine produced in the Languedoc-Roussillon region of France. The extraction procedure involved adsorption of red wine phenolics on a preparative column, alcoholic desorption and gentle evaporation of the alcoholic eluent, and spraying of the concentrated residue to obtain the polyphenol powder extract. The Provinol ${ }^{\mathrm{TM}}$ powder contains a minimum of $95 \%$ of total polyphenols (proanthocyanidols $46 \%$, prodelphinidol $21 \%$, total anthocyanins $6.1 \%$, catechin $3.8 \%$, epicatechin gallate $3 \%, \mathrm{OH}$ cinnamic acid $1.8 \%$, flavanol (quercetol) $1.4 \%$, resveratrol $0.15 \%$, free anthocyanins $0.095 \%$ ).

\section{Sampling}

Non-fasted rats were anaesthetised with pentobarbital (Ceva Santé Animale, Libourne, France) and blood was obtained from the abdominal vein with a heparinised syringe (sodium heparinate; Panpharma SA, Fougères, France). Blood samples were centrifuged at $1000 \mathrm{~g}$ for $10 \mathrm{~min}$ at $4^{\circ} \mathrm{C}$, and plasma was collected and stored at $-80^{\circ} \mathrm{C}$ until analysis. Livers were quickly removed, frozen in liquid $\mathrm{N}_{2}$ and kept at $-80^{\circ} \mathrm{C}$.

\section{Routine biochemical analyses}

Plasma glucose, total cholesterol, TAG and NEFA concentrations were measured by enzymic techniques (Konelab; Thermo Electron Corp., Vantaa, Finland). Plasma insulin and leptin levels were quantified with ELISA kits (Linco Research, St Charles, MO, USA). Protein levels in tissue homogenates were measured by Bradford's technique ${ }^{(21)}$.

Table 1. Fatty acid composition of the experimental diets $(\% \mu \mathrm{g})^{\star}$

\begin{tabular}{|c|c|c|c|}
\hline Fatty acid & Control & HFHS & $\mathrm{HFHS}+\mathrm{PPE}$ \\
\hline $8: 0$ & 0 & 0.125 & 0.266 \\
\hline $10: 0$ & 0.096 & 1.77 & $2 \cdot 17$ \\
\hline $12: 0$ & 0.135 & $23 \cdot 3$ & $26 \cdot 1$ \\
\hline $14: 0$ & 0.505 & $10 \cdot 1$ & 11 \\
\hline $16: 0$ & $12 \cdot 0$ & $10 \cdot 7$ & $10 \cdot 6$ \\
\hline $16: 1 n-7$ & 0.159 & 0.236 & 0.221 \\
\hline $18: 0$ & $3 \cdot 15$ & $7 \cdot 79$ & $7 \cdot 57$ \\
\hline $18: 1 n-9$ & 23.5 & $32 \cdot 4$ & $29 \cdot 8$ \\
\hline $18: 1 n-7$ & 1.59 & 0.820 & 0.754 \\
\hline $18: 2 n-6$ & $52 \cdot 21$ & 10.9 & $10 \cdot 2$ \\
\hline $18: 3 n-3$ & 5.55 & $1 \cdot 13$ & 1.079 \\
\hline $20: 1 n-9$ & 0.22 & 0.155 & 0.141 \\
\hline $22: 0$ & 0.64 & 0.571 & 0.187 \\
\hline $24: 0$ & 0.223 & 0 & 0 \\
\hline Total MUFA & $25 \cdot 5$ & $33 \cdot 6$ & $30 \cdot 9$ \\
\hline Total PUFA & $57 \cdot 8$ & $12 \cdot 0$ & $11 \cdot 2$ \\
\hline Total SFA & $16 \cdot 7$ & 54.4 & 57.9 \\
\hline MUFA:SFA & 1.52 & 0.618 & 0.534 \\
\hline PUFA:SFA & 3.46 & 0.221 & 0.193 \\
\hline
\end{tabular}

HFHS, high-fat-high-sucrose; PPE, polyphenol extract.

*Values are based on identifiable peaks. Each diet was analysed in duplicate. 


\section{Histological analysis}

For microscopic studies, liver samples were fixed in $10 \%$ neutral buffered formalin and embedded in paraffin. Serial tissue sections $(5 \mu \mathrm{m})$ were processed. Liver injury, such as steatosis, portal inflammatory infiltrate and fibrosis, was evaluated by histological examination after haematoxylin and eosin staining.

\section{Liver total lipid extraction and analysis}

Liver was homogenised in $\mathrm{NaCl}(9 \mathrm{~g} / \mathrm{l})$ and Triton $\mathrm{X}-100$ $(0 \cdot 1 \%)$, using an Ultra Turax homogeniser and lipids were extracted from the liver homogenate using the method of Folch et al. ${ }^{(22)}$.

NEFA, TAG and total cholesterol in the liver homogenate were quantified directly by enzymic colorimetric methods (Wako-NEFA-C kit, Oxoid, Dardilly, France; Cholesterol RTU kit, Biomerieux, Lyon, France; TG PAP kit, Biomerieux, Lyon, France) and phosphorus was quantified in the chloroform-methanol homogenate in order to determine total phospholipid quantity as previously described ${ }^{(23)}$.

The chloroform-methanol lipid extract, with 17:0 $(2500 \mu \mathrm{g} / \mathrm{ml})$ as fatty acid internal standard, was used for total fatty acid analysis by GC after transesterification.

\section{Liver total fatty acid analysis by GC}

The lipid extract was evaporated under $\mathrm{N}_{2}$ to dryness at $37^{\circ} \mathrm{C}$ and fatty acid samples were transesterified according to the method of Lepage \& Roy ${ }^{(24)}$. Briefly, the methylation reagent was generated by mixing sulfuric acid with methanol and butylated hydroxytoluene $(50 \mathrm{mg} / \mathrm{l})$, then added to the extracted dried residue and the sample was heated at $90^{\circ} \mathrm{C}$ for $45 \mathrm{~min}$ to obtain the fatty acid methyl esters. After the addition of sodium bicarbonate, distilled water and hexane, the sample was vortexed, centrifuged, and the upper hexane layer was transferred to a glass vial, evaporated under an $\mathrm{N}_{2}$ stream at $37^{\circ} \mathrm{C}$, and dissolved in iso-octane for GC analysis.

Samples were analysed on a FOCUS GC (Thermo Electron Corporation, Thermo Fisher Scientific, Courtaboeuf, France), equipped with a flame ionisation detector (Thermo Electron Corporation, Thermo Fisher Scientific). The capillary column was a TR-FAME (Thermo Electron Corporation, Thermo Fisher Scientific), $50 \mathrm{~m} \times 0.32 \mathrm{~mm}$ internal diameter with film thickness of $0.25 \mu \mathrm{m}$. The carrier gas used was He under a constant flow rate of $1.8 \mathrm{ml} / \mathrm{min}$. A $1 \mu$ l quantity of sample was injected using an AS-3000 autosampler, with a 1:6 split ratio. The temperature program was as follows: initial at $80^{\circ} \mathrm{C}$ with a $1 \mathrm{~min}$ hold; ramp: $15^{\circ} \mathrm{C} / \mathrm{min}$ to $140^{\circ} \mathrm{C}, 1^{\circ} \mathrm{C} / \mathrm{min}$ to $170^{\circ} \mathrm{C}$, and $15^{\circ} \mathrm{C} / \mathrm{min}$ to $220^{\circ} \mathrm{C}$ with a $10 \mathrm{~min}$ hold. The injector and detector were set at $220^{\circ} \mathrm{C}$. Calibration was done with fatty acid methyl ester standards from SUPELCO 37 Comp FAME Mix (SUPELCO Analytical; Sigma Aldrich, Lyon, France). Chromatograms were collected and integrated with AZUR software (Thermo Electron Corporation, Thermo Fisher Scientific). The fatty acids were quantified using the chromatographic peak area according to the internal standard method.

Detailed analysis of the fatty acid compositions both of fractions containing lipid standards and of those derived from liver was carried out by GC-MS on a Trace GC ULTRA using a Trace DSQ-MS capillary column RTx-1 $30 \mathrm{~m} \times 0.32 \mathrm{~mm}$ internal diameter $\times 0.10 \mu \mathrm{m}$ film thickness (Restek, Lisses, France) connected to a Trace DSQ mass selective detector (Thermo Fisher Scientific). Peaks were identified by comparison of electron impact ionisation spectra with a reference library.

\section{Liver desaturase activity indexes}

The activity of $\Delta 5$-desaturase, the enzyme that converts dihomo- $\gamma$-linoleic acid $(20: 3 n-6)$ to arachidonic acid $(20: 4 n-6)$, was estimated by the $20: 4 n-6 / 20: 3 n-6$ ratio $^{(25)}$. The activity of $\Delta 6$-desaturase, the enzyme that converts linoleic acid $(18: 2 n-6)$ to $\gamma$-linolenic acid $(18: 3 n-6)$, was estimated by the $18: 3 n-6 / 18: 2 n-6$ ratio $^{(26)}$. The activity of $\Delta 9$-desaturase, the enzyme that converts stearic acid $(18: 0)$ to oleic acid $(18: 1 n-9)$, was estimated by the $16: 1 n-7 /$ $16: 0$ ratio or the $18: 1 n-9 / 18: 0$ ratio $^{(26)}$. A decrease in each of these ratios can be related to a decrease of the appropriate desaturase activity and vice versa.

\section{Immunoblotting}

Frozen liver samples were homogenised using an Ultra Turax homogeniser in an ice-cold extraction buffer containing $20 \mathrm{~mm}$-2-amino-2-hydroxymethyl-propane-1,3-diol (Tris)$\mathrm{HCl}, 150 \mathrm{~mm}-\mathrm{NaCl}, 1 \mathrm{~mm}-\mathrm{EDTA}, 0.5 \%$ Triton $\mathrm{X}-100,0.1 \%$ SDS, 1 mM-phenylmethylsulfonyl fluoride, $10 \mu \mathrm{M}$-leupeptin, and $1 \mu \mathrm{M}$-pepstabtin. Proteins $(50 \mu \mathrm{g})$ were separated with 10 or $8 \%$ SDS-PAGE and then transferred to a nitrocellulose membrane (90 min; $120 \mathrm{~V})$. Membranes were blocked in $5 \%$ non-fat milk for $1 \mathrm{~h}$ at room temperature. Then, membranes were incubated overnight with primary antibodies against sirtuin-1 deacetylase (1/200; Santa Cruz Biotechnology, Santa Cruz, CA, USA), phospho-AMP kinase and AMP kinase (1/1000; Cell Signaling Technology, Inc., Danvers, MA, USA), phosphoacetyl-coA carboxylase (1/1000; Cell Signaling Technology, Inc.), fatty acid synthase (1/1000; Cell Signaling Technology, Inc.) and hepatocyte nuclear factor-4 (1/100; Santa Cruz Biotechnology) in blocking buffer. After washes in Tris buffered saline (TBS)/Tween under gentle agitation, membranes were incubated for $1 \mathrm{~h}$ with horseradish peroxidase-labelled antibody (1/5000 for Santa Cruz antibody or 1/2000 for Cell Signaling antibody). After further washes, blots were treated with enhanced chemiluminescence detection reagents (ECL, ThermoScientific). $\beta$-Actin was used as a loading reference, and blot intensities were measured using the BIO-Profil 1D software (Fisher Bioblock, Illkirch, France).

\section{Liver mRNA expression}

Real-time quantitative PCR (RT-qPCR) was used to measure target gene mRNA expression in liver. Total RNA was extracted with Trizol reagent (Invitrogen Life Technologies, Cergy Pontoise, France). Reverse transcription reaction was performed with $5 \mu \mathrm{g}$ total RNA. cDNA was synthesised with the use of SuperScript II RT for first-strand cDNA synthesis (Invitrogen Life Technologies, Cergy Pontoise, France) and Oligo (dT) primers. The mRNA expressions of target genes were determined by RT-qPCR. RT-qPCR analysis 
was performed using IQ ${ }^{\mathrm{TM}}$ SYBR Green Supermix (Biorad, Hercules, CA, USA) with a MiniOpticon detection system (Biorad, Hercules, CA, USA). Results were normalised with the gene encoding $18 \mathrm{~S}$ used as the reference. The primer sequences used for real-time RT-PCR are shown in Table 2. After normalisation by $18 \mathrm{~S}$, all results are expressed as percentage of control as mean values and standard deviations.

\section{Liver mitochondrial respiratory complex activities}

Liver homogenates were prepared on ice in a ratio of $1 \mathrm{~g}$ wet tissue for $9 \mathrm{ml}$ phosphate buffer $(50 \mathrm{~mm}$; pH 7) using a Polytron homogeniser and centrifuged at $1000 \mathrm{~g}$ for $10 \mathrm{~min}$ at $4^{\circ} \mathrm{C}$. Complex II and complex II + III activities in supernatant fraction were determined spectrophotometrically according to Rustin et al. ${ }^{(27)}$. Complex IV or cytochrome c oxidase activity was measured spectrophotometrically according to Wharton \& Tzagoloff ${ }^{(28)}$. Citrate synthase activity was measured spectrophotometrically according to Srere $^{(29)}$.

\section{Statistical analysis}

Results were expressed as mean values and standard deviations. Statistical analyses were based on one-way ANOVA followed by Fisher's multiple-comparisons test. The limit of statistical significance was set at $P<0 \cdot 05$. Statistical analyses were performed using the StatView program (SAS Institute, Cary, NC, USA).

\section{Results}

Characterisation of high-fat-high-sucrose-fed rats and high-fat-high-sucrose plus polyphenols-fed rats

Weight gain by kJ of diet was significantly higher in rats fed the HFHS diet and the HFHS + PPE diet, but no difference was observed between the HFHS group and the HFHS + PPE group. The body weight of rats fed the HFHS diet and the HFHS + PPE diet was higher, but not significantly, than that of rats fed the control diet, whereas liver weight was significantly higher (Tables 3 and 4). However, the liver weight:body weight ratio was similar among groups (Table 4). The mean dietary intake and energy intake during the experiment were not significantly modified among groups (Table 3 ).

Plasma TAG, total cholesterol and NEFA levels were not significantly different among control, HFHS and HFHS +
PPE rats, even if plasma TAG and total cholesterol increased by about $10-15 \%$ in HFHS rats, showing a great interindividual variation (Table 3). Plasma glucose level was increased with the HFHS and HFHS + PPE diets in comparison with the control diet. Plasma insulin level was increased, but not significantly, in HFHS rats in comparison with control rats and significantly decreased with the HFHS-PPE diet in comparison with HFHS rats. Plasma leptin level was significantly increased in rats fed the HFHS diet and the HFHS + PPE diet in comparison with the control diet (Table 3).

Visual observation of liver from HFHS rats revealed a yellow macroscopic appearance, suggesting fatty change but less important in livers from HFHS + PPE rats and not in control rats. The histological features in the liver of control (Fig. 1(a)), HFHS (Fig. 1(b)) and HFHS + PPE (Fig. 1(c)) diet-fed rats were hepatic macro-steatosis with HFHS, recognisable by a preponderance of large droplets in which a single, bulky fat vacuole distends the hepatocyte and pushes the nucleus and cytoplasm to the side and microvacuolar steatosis with HFHS + PPE, with small intracytoplasmic droplets. In the control group, no steatosis was observed. This suggests that induction of hepatic steatosis by the HFHS diet was partially prevented by polyphenol administration.

Liver TAG, total cholesterol and phospholipid levels were higher in HFHS- and HFHS + PPE-fed rats in comparison with control rats (Table 4). Liver NEFA levels were not different among control, HFHS and HFHS + PPE rats. Moreover, hepatic TAG content was significantly reduced in rats fed the HFHS + PPE diet in comparison with the HFHS diet, which was consistent with the observed histological changes (Fig. 1).

\section{Total fatty acid composition of liver, desaturase activity indexes and stearoyl-CoA desaturase $1 \mathrm{mRNA}$ expression}

Total major SFA, MUFA and PUFA were in higher quantity in the liver of rats fed the HFHS and HFHS + PPE diets than in those fed the control diet (Table 5). In animals fed the HFHS + PPE diet, the PPE did not influence significantly the total fatty acids profile: total SFA, MUFA and PUFA quantities were not modified in comparison with rats fed the HFHS diet; surprisingly, total $n-3$ PUFA quantities, and in particular 22:6n-3 (DHA), were significantly lower in rats fed the HFHS + PPE diet in comparison with rats fed the HFHS diet (Table 5). The $n-6: n-3$ ratio did not significantly

Table 2. Primer sequences used for real-time RT-PCR

\begin{tabular}{lll}
\hline & Forward & Reverse \\
\hline Sirtuin-1 deacetylase & AAC TTC ACA GCA TCT TCA ATT GTA TT & TGA CAC TGT GGC AGA TTG TTA TT \\
PGC-1 $\alpha$ & ATG TGT CGC CTT CTT GCT CT & ATC TAC TGC CTG GGG ACC TT \\
Sterol regulatory element-binding protein-1c & GGA GCC ATG GAT TGC ACA TT & AGG AAG GCT TCC AGA GAG GA \\
Carnitine palmitoyltransferase 1 & TCT TGC AGT CGA CTC ACC TT & TCC ACA GGA CAC ATA GTC AGG \\
Malonyl-CoA decarboxylase & CGG CAC CTT CCT CAT AAA GC & GGG TAT AGG TGA CAG GCT GGA \\
Medium-chain acyl-CoA dehydrogenase & CTC CGC AGA GGC TAC AAG & TGA TGA GAG GGA ACG GGT AT \\
Nuclear respiratory factor 1 & TTA TTC TGC TGT GGC TGA TGG & CCT CTG ATG CTT GCG TCG TCT \\
Mitochondrial transcription factor A & GCT AAA CAC CCA GAT GCA AAA & CGA GGT CTT TTT GGT TTT CC \\
SCD1 & GAA GAA GGT GCC CCT CTA TCT & AGA GCA AGG TGT AGA CCT TGG \\
$18 S$ & GAG GTG AAA TTC TTG GAC CGG & CGA ACC TCC GAC TTT CGT TCT \\
\hline
\end{tabular}

PGC-1 $\alpha$, PPAR $\gamma$ coactivator-1 $\alpha$; SCD1, stearoyl-CoA desaturase 1. 
Table 3. Body weight and weight gain, dietary and energy intakes and plasma glucose, lipids, insulin and leptin levelsł

(Mean values and standard deviations)

\begin{tabular}{|c|c|c|c|c|c|c|}
\hline \multirow{2}{*}{ Diet... } & \multicolumn{2}{|c|}{ Control } & \multicolumn{2}{|c|}{ HFHS } & \multicolumn{2}{|c|}{$\mathrm{HFHS}+\mathrm{PPE}$} \\
\hline & Mean & SD & Mean & SD & Mean & SD \\
\hline Weight gain (g/kJ diet) & 0.0060 & 0.014 & $0.0098^{*}$ & 0.0024 & $0.0089^{*}$ & 0.0024 \\
\hline Final body weight $(\mathrm{g})$ & 509 & 52 & 583 & 63 & 572 & 91 \\
\hline Dietary intake $(\mathrm{g} / \mathrm{d})$ & 21.5 & $2 \cdot 0$ & 17.5 & $2 \cdot 0$ & 18.5 & 3.2 \\
\hline Energy intake $(\mathrm{kJ} / \mathrm{d})$ & 342 & 31 & 372 & 43 & 393 & 67 \\
\hline Glucose (mmol/l) & $10 \cdot 1$ & $1 \cdot 0$ & $12 \cdot 2^{*}$ & $1 \cdot 1$ & $11.9^{*}$ & 1.4 \\
\hline Cholesterol $(\mathrm{mmol} / \mathrm{l})$ & $2 \cdot 02$ & 0.34 & $2 \cdot 20$ & 0.38 & $2 \cdot 32$ & 0.54 \\
\hline $\mathrm{TAG}(\mathrm{mmol} / \mathrm{l})$ & $2 \cdot 01$ & 0.21 & $2 \cdot 16$ & 1.40 & 2.93 & 1.09 \\
\hline NEFA (mmol/l) & 0.277 & 0.142 & 0.240 & 0.066 & 0.366 & 0.119 \\
\hline Insulin ( $\mu \mathrm{g} / \mathrm{l})$ & $7 \cdot 19$ & 1.85 & $8 \cdot 84$ & 1.00 & $6 \cdot 21 \dagger$ & 1.28 \\
\hline Leptin $(\mu \mathrm{g} / \mathrm{l})$ & $13 \cdot 1$ & $6 \cdot 8$ & $19 \cdot 4$ & 4.4 & $19 \cdot 9$ & 4.0 \\
\hline
\end{tabular}

HFHS, high-fat-high-sucrose; PPE, polyphenol extract.

${ }^{*}$ Mean value was significantly different from that of the control group $(P<0.05)$

† Mean value was significantly different from that of the HFHS group $(P<0.05)$.

$\ddagger$ One-way ANOVA was used followed by Fisher's multiple-comparisons test. Significance was set at $P<0.05$.

vary with diet, suggesting that the HFHS diet or the PPE did not influence the balance of these essential PUFA and highly unsaturated fatty acids (Table 5).

The 18:1n-9/18:0 ratio, a $\Delta 9$-desaturase activity index, was in higher proportion in the liver of rats fed the HFHS and HFHS + PPE diets than in those fed the control diet, and the PPE did not modify it; in contrast the $16: 1 n-9 / 16: 0$ ratio, also a $\Delta 9$-desaturase activity index, was in lower proportion. The $\Delta 6$-desaturase activity index was increased and the $\Delta 5$-desaturase activity index was decreased in rats fed the HFHS diet and the HFHS + PPE diet in comparison with controls (Table 5).

There was a trend towards lower $S C D 1$ mRNA expression in the liver of rats fed the HFHS (0.416 (SD 0.404)) or the HFHS + PPE (0.462 (SD 0.283)) diet in comparison with controls $(1.00$ (SD 0.75$))(P=0.0716$ for HFHS $v$. controls and $P=0.0944$ for HFHS + PPE $v$. controls).

\section{Sirtuin-1 deacetylase-AMP kinase signalling pathway}

Sirtuin-1 deacetylase protein expression level was significantly increased in rats fed the HFHS + PPE diet in comparison with controls and HFHS-fed animals (Fig. 2), while sirtuin-1 deacetylase mRNA expression level was unchanged (data not shown).

Total AMP kinase protein expression level was decreased, but not significantly, in rats fed the HFHS diet in comparison with controls, than significantly increased to control values in rats fed the HFHS + PPE diet in comparison with those fed the HFHS diet, while phosphorylated AMP kinase expression level was unchanged, as was phospho-AMP kinase:AMP kinase (Fig. 2).

Phosphorylated acetyl-CoA carboxylase protein expression level was significantly decreased in rats fed the HFHS diet in comparison with controls and returned to control values in rats fed the HFHS + PPE diet (Fig. 2).

Table 4. Liver weight and lipid content, and mitochondrial respiratory complex activitiesł (Mean values and standard deviations)

\begin{tabular}{|c|c|c|c|c|c|c|}
\hline \multirow[t]{2}{*}{ Diet... } & \multicolumn{2}{|c|}{ Control } & \multicolumn{2}{|c|}{ HFHS } & \multicolumn{2}{|c|}{ HFHS + PPE } \\
\hline & Mean & SD & Mean & SD & Mean & SD \\
\hline Liver weight (g) & $16 \cdot 4$ & $2 \cdot 2$ & $19 \cdot 8^{*}$ & $2 \cdot 2$ & $19 \cdot 1^{*}$ & $2 \cdot 4$ \\
\hline Liver relative weight (\%) & $3 \cdot 2$ & 0.3 & 3.4 & 0.2 & 3.4 & 0.4 \\
\hline NEFA (mg/g wet weight) & 5.02 & 1.06 & 5.40 & 0.80 & 5.54 & 1.35 \\
\hline Cholesterol (mg/g wet weight) & $2 \cdot 64$ & 0.37 & $4 \cdot 26^{\star}$ & 0.93 & $4 \cdot 33^{*}$ & 1.43 \\
\hline Phospholipids (mg/g wet weight) & $25 \cdot 9$ & 1.4 & $28 \cdot 6^{\star}$ & 1.4 & $28 \cdot 6^{*}$ & $2 \cdot 6$ \\
\hline TAG (mg/g wet weight) & $20 \cdot 2$ & $3 \cdot 3$ & $60 \cdot 5^{\star}$ & $12 \cdot 3$ & $46 \cdot 1^{*} \dagger$ & 11.4 \\
\hline CS (mU/mg protein) & 55.5 & 3.4 & $56 \cdot 1$ & $6 \cdot 6$ & $59 \cdot 2$ & 6.7 \\
\hline Complex II (mU/mg protein) & 93.0 & $7 \cdot 0$ & $72 \cdot 8^{\star}$ & $18 \cdot 1$ & $80 \cdot 3$ & 14.5 \\
\hline Complex II:CS & 1.68 & 0.18 & $1.30^{*}$ & 0.30 & $1.36^{*}$ & 0.24 \\
\hline Complex II + III (mU/mg protein) & 6.43 & 0.94 & $4.83^{*}$ & 0.67 & $5 \cdot 40^{*}$ & 0.89 \\
\hline Complex II + III:CS & $0 \cdot 117$ & 0.021 & $0.086^{*}$ & 0.007 & $0.092^{*}$ & 0.018 \\
\hline Complex IV (mU/mg protein) & $52 \cdot 2$ & $12 \cdot 3$ & $51 \cdot 6$ & $12 \cdot 1$ & $58 \cdot 9$ & $14 \cdot 0$ \\
\hline Complex IV:CS & 0.949 & 0.249 & 0.920 & 0.176 & 0.992 & 0.195 \\
\hline
\end{tabular}

HFHS, high-fat-high-sucrose; PPE, polyphenol extract; CS, citrate synthase; complex IV, cytochrome c oxidase.

${ }^{*}$ Mean value was significantly different from that of the control group $(P<0.05)$.

† Mean value was significantly different from that of the HFHS group $(P<0.05)$.

$\ddagger$ One-way ANOVA was used followed by Fisher's multiple-comparisons test. Significance was set at $P<0.05$. 
(a)

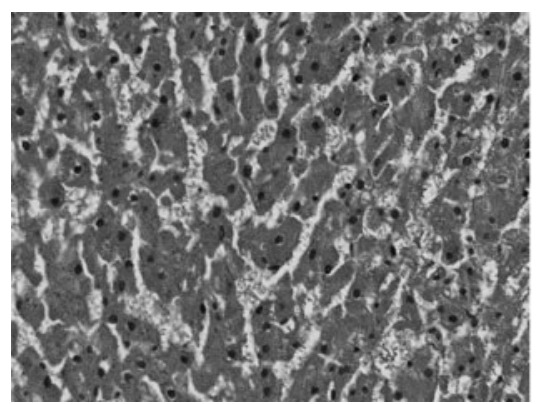

(b)

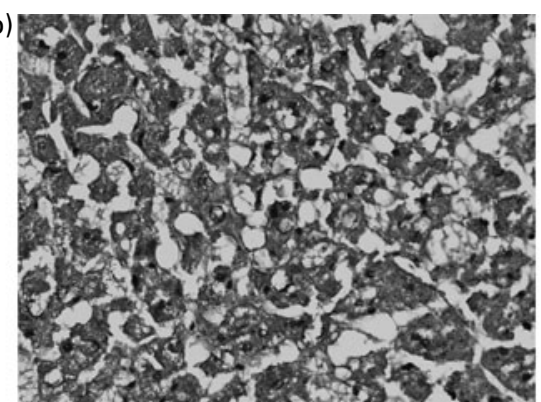

(c)

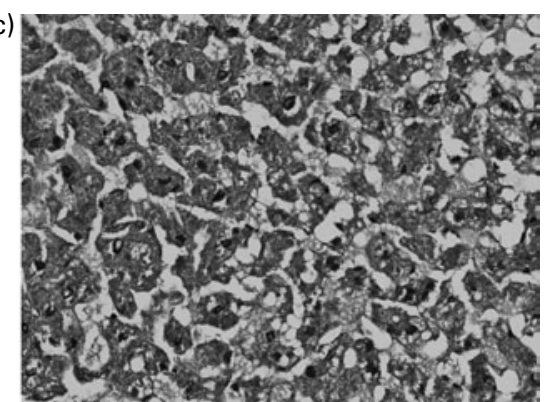

Fig. 1. Liver histology after haematoxylin-eosin staining of liver sections from a representative rat of each group: (a) control diet; (b) high-fat-high-sucrose (HFHS) diet; (c) HFHS diet plus polyphenol extract.

Phospho-acetyl-CoA carboxylase protein expression was almost negatively correlated with liver TAG content $(r-0.474$; $P=0.0867)$.

Sterol regulatory element-binding protein-1c (SREBP-1c) mRNA expression level was unchanged (data not shown). Fatty acid synthase and hepatocyte nuclear factor-4 protein expression levels were not different among control, HFHS and HFHS + PPE rats (Fig. 2).

(Mean values and standard deviations)
PPAR $\gamma$ coactivator- $1 \alpha(P G C-1 \alpha)$ mRNA expression level was significantly decreased in rats fed the HFHS diet in comparison with controls, and $P G C-1 \alpha$ mRNA expression level in rats fed the HFHS + PPE diet was increased in comparison with HFHS diet-fed rats but not significantly (1.00 (SD 0.43), 0.387 (SD 0.123), 0.708 (SD 0.525), for control, HFHS and HFHS + PPE, respectively). $P G C-1 \alpha$ gene expression was negatively correlated with liver TAG content $(r-0 \cdot 608 ; P<0 \cdot 05)$.

Table 5. Total fatty acid composition of rat liver ( $\mathrm{mg}$ fatty acid/g tissue)‡

\begin{tabular}{|c|c|c|c|c|c|c|}
\hline \multirow[t]{2}{*}{ Diet... } & \multicolumn{2}{|c|}{ Control } & \multicolumn{2}{|c|}{ HFHS } & \multicolumn{2}{|c|}{ HFHS + PPE } \\
\hline & Mean & SD & Mean & SD & Mean & SD \\
\hline Total SFA & 14.5 & 1.61 & $25 \cdot 8^{\star}$ & 4.78 & $23 \cdot 6^{*}$ & 4.57 \\
\hline $12: 0$ & 0.079 & 0.059 & $0.638^{*}$ & 0.244 & $0.490^{\star}$ & 0.196 \\
\hline $14: 0$ & 0.476 & 0.097 & $2 \cdot 42^{*}$ & 0.802 & $2 \cdot 07^{\star}$ & 0.601 \\
\hline $16: 0$ & $9 \cdot 10$ & 1.28 & $14 \cdot 9^{*}$ & $3 \cdot 26$ & $13 \cdot 8^{*}$ & $2 \cdot 76$ \\
\hline $18: 0$ & 4.69 & 0.751 & $7 \cdot 36^{\star}$ & 1.05 & $6 \cdot 94^{\star}$ & 1.07 \\
\hline Total MUFA & $14 \cdot 7$ & 4.05 & $34.6^{*}$ & 9.49 & $29 \cdot 6^{\star}$ & $6 \cdot 13$ \\
\hline $16: 1 n-9$ & 0.178 & 0.037 & $0.433^{\star}$ & 0.094 & $0.429^{\star}$ & 0.115 \\
\hline $16: 1 n-7$ & $2 \cdot 13$ & 0.955 & $1 \cdot 23^{\star}$ & 0.619 & $1 \cdot 20^{*}$ & 0.347 \\
\hline $18: 1 n-9$ & 5.93 & 1.36 & $27 \cdot 7^{\star}$ & 7.56 & $23 \cdot 3^{*}$ & $6 \cdot 28$ \\
\hline $18: 1 n-7$ & $2 \cdot 17$ & 0.588 & $2 \cdot 13$ & 0.467 & 1.91 & 0.237 \\
\hline $20: 1 n-9$ & 0.009 & 0.013 & $0.436^{\star}$ & 0.129 & $0.308^{\star} \dagger$ & 0.107 \\
\hline Total PUFA & $12 \cdot 7$ & 1.60 & $24 \cdot 3^{*}$ & 4.72 & $20 \cdot 3^{\star}$ & 3.87 \\
\hline $18: 2 n-6$ & $5 \cdot 19$ & $1 \cdot 17$ & $12 \cdot 8^{*}$ & $3 \cdot 27$ & $10 \cdot 3^{*}$ & $2 \cdot 72$ \\
\hline $18: 3 n-6$ & 0.038 & 0.017 & $0 \cdot 141^{*}$ & 0.069 & $0.148^{*}$ & 0.074 \\
\hline $18: 3 n-3$ & 0.195 & 0.089 & $0.664^{\star}$ & 0.262 & $0.481^{*}$ & 0.139 \\
\hline $20: 2 n-6$ & 0.008 & 0.007 & $0.210^{*}$ & 0.088 & $0.132^{\star} \dagger$ & 0.056 \\
\hline $20: 3 n-6$ & 0.233 & 0.063 & $0.861^{*}$ & 0.370 & $0.664^{\star}$ & 0.175 \\
\hline $20: 4 n-6$ & $5 \cdot 33$ & 0.523 & $6 \cdot 56$ & 0.720 & $6 \cdot 15$ & 0.819 \\
\hline $20: 5 n-3$ & n.d. & n.d. & n.d. & n.d. & n.d. & n.d. \\
\hline $22: 4 n-6$ & 0.072 & 0.031 & $0.394^{\star}$ & $0 \cdot 101$ & $0.253^{\star} \dagger$ & 0.147 \\
\hline $22: 5 n-3$ & 0.117 & 0.071 & $0.366^{*}$ & 0.225 & 0.262 & 0.157 \\
\hline $22: 6 n-3$ & 1.55 & 0.162 & $2 \cdot 27^{\star}$ & 0.363 & $1.89^{*} \dagger$ & 0.300 \\
\hline Total fatty acids & $37 \cdot 7$ & 4.75 & $82 \cdot 1^{*}$ & $17 \cdot 8$ & $71 \cdot 1^{*}$ & 14.8 \\
\hline Total unsaturated fatty acids & $23 \cdot 0$ & $3 \cdot 22$ & $55 \cdot 6^{*}$ & $12 \cdot 8$ & $47 \cdot 0^{*}$ & $10 \cdot 0$ \\
\hline Total $n-6$ & $10 \cdot 9$ & 1.48 & $21 \cdot 0^{*}$ & 3.96 & $17 \cdot 6^{\star}$ & 3.48 \\
\hline Total $n-3$ & 1.86 & 0.171 & $3 \cdot 32^{\star}$ & 0.770 & $2 \cdot 63^{*} \dagger$ & 0.430 \\
\hline$n-6: n-3$ & 5.85 & 0.626 & $6 \cdot 37$ & 0.448 & $6 \cdot 70^{\star}$ & 0.660 \\
\hline \multicolumn{7}{|l|}{ Desaturase activity index } \\
\hline$\Delta 9(16: 1 n-7 / 16: 0)$ & 0.227 & 0.077 & $0.080^{*}$ & 0.020 & $0.094^{\star}$ & 0.045 \\
\hline$\Delta 9(18: 1 n-9 / 18: 0)$ & $1 \cdot 272$ & 0.244 & $3.76^{\star}$ & 0.781 & $3 \cdot 31^{\star}$ & 0.492 \\
\hline$\Delta 6(18: 3 n-6 / 18: 2 n-6)$ & 0.007 & 0.002 & 0.011 & 0.003 & $0.013^{*}$ & 0.004 \\
\hline$\Delta 5(20: 4 n-6 / 20: 3 n-6)$ & $24 \cdot 0$ & $5 \cdot 34$ & $8 \cdot 71^{*}$ & 3.46 & $9 \cdot 93^{\star}$ & $3 \cdot 14$ \\
\hline
\end{tabular}

HFHS, high-fat-high-sucrose; PPE, polyphenol extract; n.d., non-detectable.

${ }^{*}$ Mean value was significantly different from that of the control group $(P<0.05)$.

† Mean value was significantly different from that of the HFHS group $(P<0.05)$.

$\ddagger$ One-way ANOVA was used followed by Fisher's multiple-comparisons test. Significance was set at $P<0.05$. 


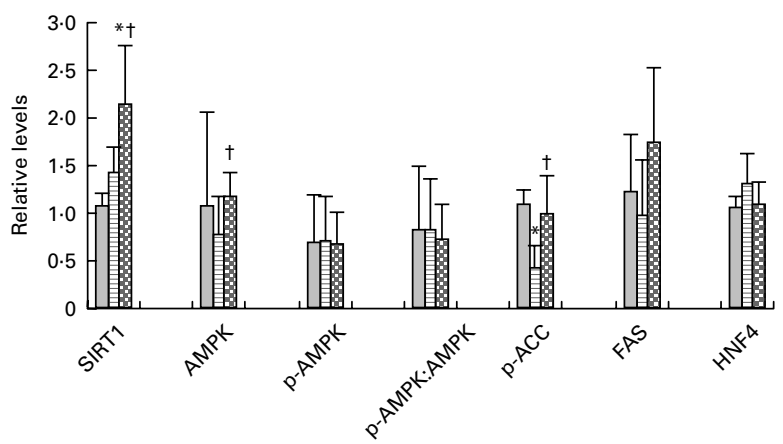

Fig. 2. Sirtuin-1 deacetylase (SIRT1)-AMP kinase (AMPK) signalling pathway: relative protein expression in control diet-fed rats $(\square)$, high-fat-highsucrose (HFHS) diet-fed rats (日) and HFHS diet plus polyphenol extract-fed rats (国). p-AMPK, phospho-AMPK; p-ACC, phospho-acetyl-CoA carboxylase; FAS, fatty acid synthase; HNF4, hepatocyte nuclear factor 4. Values are means ( $n 6$ per group), with standard deviations represented by vertical bars. * Mean value was significantly different from that of the control group $(P<0.05)$. †Mean value was significantly different from that of the HFHS group $(P<0.05)$.

Carnitine palmitoyltransferase 1, malonyl-CoA decarboxylase, medium-chain acyl-CoA dehydrogenase, nuclear respiratory factor 1 and mitochondrial transcription factor $\mathrm{A}$ mRNA expression levels were not different among control, HFHS and HFHS + PPE rats (data not shown).

\section{Mitochondrial respiratory complex activities}

Liver complex II and complex II + III activities and complex II:citrate synthase and complex II + III:citrate synthase ratios were significantly decreased in rats fed the HFHS and HFHS + PPE diets in comparison with controls (Table 4). No significant difference was observed between rats fed the HFHS or the HFHS + PPE diet. Citrate synthase and complex IV activities were not different among control, HFHS and HFHS + PPE rats. Complex IV activity and complex IV:citrate synthase were significantly correlated with liver NEFA content $(r 0.615, P<0.01$ and $r 0.505, P<0.05$, respectively), while complex II + III activity, complex II + III:citrate synthase and complex II:citrate synthase were negatively correlated with liver TAG content $(r-0.558, P<0.05$, $r-0.666, P<0.01$ and $r-0.611, P<0.01$, respectively).

\section{Discussion}

Fatty liver, the initial stage of NAFLD, is characterised by an increased content of hepatocellular lipids. Our study was conducted to determine the effects of a PPE on both quantity and quality of liver lipids in HFHS diet-fed rats. The PPE was given mixed to the diet at a concentration that provided about $30 \mathrm{mg}$ polyphenols $/ \mathrm{kg}$ per $\mathrm{d}$, which is only two-fold the estimated dietary intake in humans ${ }^{(30)}$.

\section{Characteristics of rats}

HFHS diet-fed rats gained significantly more weight than control rats and their plasma leptin level, among the bestknown hormone marker for obesity ${ }^{(31)}$, was also increased. The supplementation of rats fed the HFHS + PPE diet did not modify significantly either weight gain or plasma leptin level, probably because the duration of the supplementation was too short to obtain a significant beneficial effect of this PPE. In fact, a potentially preventive effect on diet-induced obesity of polyphenol consumption has been described for a longer period of supplementation ${ }^{(32-34)}$. It cannot be excluded nevertheless that the lack of effect was due to the different model (HFHS) used to induce fat accumulation in the liver with respect to the most common model of a high-fat diet, used in the studies cited.

Plasma glucose was significantly increased in the HFHS diet-fed rats by comparison with control rats. Moreover, insulin levels, plasma TAG and cholesterol tended to increase but non-significantly in the HFHS diet-fed rats compared with the control group, and plasma NEFA were not modified by the HFHS diet. Thus, the model of NAFLD used here (HFHS diet) seems to induce a very early stage of liver steatosis, as it lacks most of the hallmarks associated with NAFLD such as fatty acid increase, hypertriacylglycerolaemia and hyperinsulinaemia, usually observed with a high-fat diet. PPE intake modulated slightly this model by decreasing insulin levels in comparison with the HFHS group, but it failed to affect plasma glucose levels.

\section{Liver steatosis and total lipid content}

Liver macrosteatosis was observed in HFHS diet-fed rats and liver microsteatosis, the first step to liver macrosteatosis, was observed in the HFHS + PPE group but not in the control group. Insulin resistance is currently considered as the basis for intrahepatic lipid accumulation ${ }^{(35)}$. However, insulinaemia was not significantly increased in the HFHS diet-fed rats with liver steatosis. It was suggested recently that hepatic steatosis is not necessarily associated with insulin resistance and that accumulation of intrahepatic lipids may precede the state of insulin resistance, while hepatic TAG itself may not be toxic and may in fact protect the liver from lipotoxicity by buffering the accumulation of fatty acids ${ }^{(36)}$.

In accordance with visual observation of liver steatosis, liver TAG content was increased in HFHS diet-fed rats and this increase was less important with PPE supplementation. Thus, the PPE partially prevented the accumulation of TAG in the liver, as previously observed with other polyphenols $^{(37-40)}$.

\section{Liver fatty acid composition}

Dietary lipids influenced the rat liver fatty acid composition, as SFA and MUFA, but not PUFA, were found in the same proportion in diets and in rat livers. Similar results were also observed in fatty composition of the different lipid subclasses of phospholipids, TAG, cholesteryl ester and NEFA (data not shown). These results suggest that liver lipid homeostasis is modulated by dietary fatty acids. Concerning PUFA, the dietary fatty acid levels of $18: 2 n-6$ and $18: 3 n-3$ were 5-fold lower in the HFHS diet but significantly higher in the liver of rats fed the HFHS and HFHS + PPE diets in comparison with control rats. These fatty acids may be stored or metabolically converted to highly unsaturated fatty acids $(20: 4 n-6,20: 5 n-3 \text { and } 22: 6 n-3)^{(41)}$, and as the $20: 4 n-6$ content was not significantly different in the livers of rats fed the different diets, there was probably suppression 
of the conversion of $18: 2 n-6$ PUFA to $20: 4 n-6$ highly unsaturated fatty acid and net accumulation of $18: 2 n-6$ from the diet in the liver of rats fed the HFHS diets. The $18: 3 n$ 3 content was very low and $20: 5 n-3$ content was undetectable in rats fed the different diets, suggesting a nearly $100 \%$ conversion of these $n-3$ fatty acids to $22: 6 n-3$. Surprisingly, the $22: 6 n-3$ level increased in the liver of rats fed the HFHS or HFHS + PPE diet in comparison with controls, although the proportion of $18: 3 n-3$, precursor of $22: 6 n-3$, was 5-fold higher in the control diet than in the HFHS diets. Such an effect of $18: 3 n-3$ conversion into its long-chain derivatives such as DHA has previously been found ${ }^{(42)}$. Moreover, 22:6n-3 and n-3 PUFA contents decreased in the liver of rats fed the HFHS + PPE diet in comparison with the HFHS diet, but we have no explanation for this observation.

SCD1 is a microsomal enzyme that catalyses the synthesis of monounsaturated long-chain fatty acids from saturated fatty acyl-CoA ${ }^{(26)}$, and its preferred substrates are palmitoyl$(16: 0)$ and stearoyl-CoA $(18: 0)$. The $18: 1 n-9 / 18: 0$ ratio, which is usually used to express $\Delta 9$-desaturase activity ${ }^{(26)}$, was higher in rats fed the HFHS diets in comparison with controls, with no effect of PPE observed. However, this ratio probably did not express the real activity of $\Delta 9$-desaturase in the present study, because $18: 1 n-9$ and $18: 0$ were in higher proportion in the HFHS diets than in the control diet. On contrary, the $16: 1 n-7 / 16: 0$ ratio was lower in rats fed the HFHS diets in comparison with controls, and as the control and HFHS diets provided the same proportion of these two fatty acids, this result also suggested a decreased $\Delta 9$-desaturase activity. In accordance, $S C D 1$ gene expression decreased in rats fed the HFHS or HFHS + PPE diet in comparison with controls, but unlike what was described in mice fed a high-fat diet ${ }^{(43)}$. Also, no effect of the PPE was observed on SCD1 gene expression, unlike previously described ${ }^{(12,13)}$. It is possible that the high intake of $18: 1 n-9$ in rats fed the HFHS diets suppressed de novo 18:1n-9 MUFA synthesis. $\Delta 6$-Desaturase and $\Delta-5$ desaturase activity indexes were modulated according to the quantity of lipids in the diet, with no effect of the PPE.

\section{The sirtuin-1 deacetylase-AMP kinase signalling pathway}

Sirtuin-1 deacetylase and AMP kinase are two critical signalling molecules controlling the pathways of hepatic lipid metabolism. Resveratrol and other polyphenols have been identified as potent agonists of sirtuin-1 deacetylase ${ }^{(44)}$ and several lines of investigation have demonstrated that resveratrol modulates lipid metabolism mainly through activation of sirtuin-1 deacetylase signalling ${ }^{(6,45,46)}$. In the present study and in agreement with such observations, the expression of sirtuin-1 deacetylase protein increased significantly in the liver of rats fed the HFHS + PPE diet in comparison with rats fed the HFHS and control diets.

Although many other factors may contribute to the hepatocyte metabolic effects of sirtuin-1 deacetylase, sirtuin-1 deacetylase activation by polyphenols may stimulates AMP kinase $^{(16)}$. In the present study, the expression of total AMP kinase was increased in HFHS + PPE diet-fed rats in comparison with HFHS-fed rats. However, the expression of phosphorylated AMP kinase, the active form, did not change whatever the administered diet; this was probably linked to the great variability observed within the same group and the transitory phenomenon of protein phosphorylation.

Activated acetyl-CoA carboxylase is a key enzyme that plays a key role in the regulation of fatty acid metabolism, by modulating lipid synthesis and lipid catabolism in mitochondria $^{(15)}$. In the present study, the expression of phosphorylated acetyl-CoA carboxylase (inactive form) decreased in HFHS diet-fed rats in comparison with controls, and was restored to control values in rats fed the HFHS + PPE diet. It was almost significantly correlated to the liver TAG content, underlying its central role in liver lipid accumulation. In agreement with the present results, previous studies have also demonstrated that acetyl-CoA carboxylase is up-regulated in the obese state and in response to highcarbohydrate diets ${ }^{(47,48)}$ while it is down-regulated by various polyphenols $^{(15,16,37,49)}$.

SREBP-1c is a transcriptional factor that activates the expression of key enzymes involved in lipogenesis ${ }^{(7,50)}$. Moreover, hepatocyte nuclear factor-4, a downstream target of AMP kinase ${ }^{(51)}$, is essential for the maintenance of lipid homeostasis $^{(52)}$. In the present study, the gene expression of SREBP1-c was unchanged among the three groups. Moreover, the protein expression of hepatocyte nuclear factor- 4 was not modified whatever the diet. The amount of protein fatty acid synthase, a target of SREBP1-c, that plays a central role in de novo lipogenesis, was also unchanged ${ }^{(53)}$. Thus, it was probably not SREBP1-c-inhibited lipogenesis that contributes to lower hepatic TAG accumulation with polyphenols.

Sirtuin-1 deacetylase has been shown to modulate PGC- $1 \alpha$ activity $^{(46,54)}$ while PGC- $1 \alpha$ increases the oxidation of fatty acids via increasing mitochondrial capacity and function ${ }^{(55)}$. In the present study, the gene expression of $P G C-1 \alpha$ decreased in HFHS diet-fed rats in comparison with controls and returned almost to control values with the PPE, as previously described with resveratrol ${ }^{(12,45)}$. Moreover, $P G C-1 \alpha$ gene expression was negatively correlated with liver TAG content. Nevertheless, the expression of its target genes carnitine palmitoyltransferase 1 and medium-chain acyl-CoA dehydrogenase, that regulate mitochondrial fatty acid $\beta$-oxidation ${ }^{(56)}$, was not modified whatever the diet. It would be interesting to explore whether the PPE rather modulates their activities. Thus, PPE may attenuate fatty liver induced by the HFHS diet through the activation of hepatic sirtuin-1 deacetylase, and by the regulation of acetyl-CoA carboxylase phosphorylation. Nevertheless, we cannot exclude that the PPE may exert part of its hypolipidaemic effect by inhibiting the intestinal absorption of dietary lipids and diminishing chylomicron secretion by enterocytes, as it has been demonstrated for green tea catechins ${ }^{(57,58)}$. This hypothesis deserves further study, as this has never been addressed for wine polyphenols.

\section{Mitochondrial biogenesis and mitochondrial activity}

Mitochondria are the principal energy sources of the cell that convert nutrients into energy through cellular respiration ${ }^{(59)}$, and mitochondrial biogenesis in the liver is controlled, in large part, by the transcriptional coactivator PGC- $1 \alpha^{(6,54)}$. Thus, we have explored $P G C-1 \alpha$ target mitochondrial genes and maximal activities of mitochondrial respiratory chain complexes. The gene expression of $P G C-1 \alpha$ was 


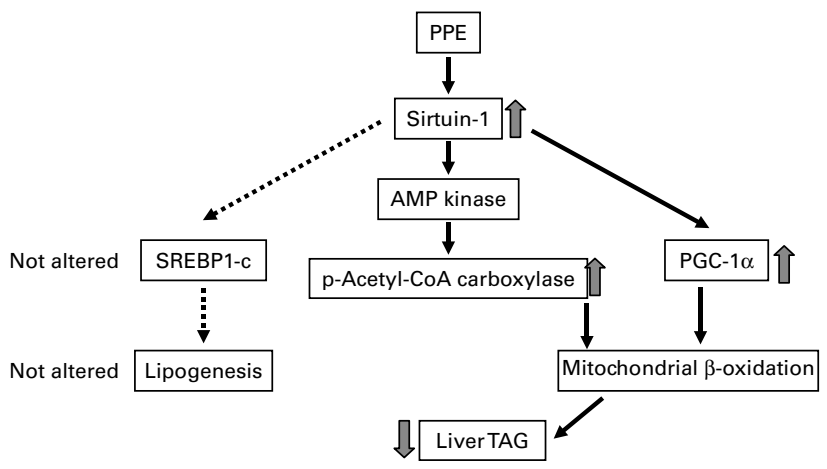

Fig. 3. Explanatory diagram showing the pathways found to be affected by polyphenol extract (PPE). As shown, PPE activates hepatic sirtuin-1 deacetylase. This latter activates directly or indirectly PPAR $\gamma$ coactivator- $1 \alpha$ (PGC-1 $\alpha$ ) and inactivates acetyl-CoA carboxylase by enhancing its phosphorylation via AMP kinase. Both PGC-1 $\alpha$ and acetyl-CoA carboxylase may contribute to increase mitochondrial fatty acid oxidation. On the other hand, PPE does not alter sterol regulatory element-binding protein-1c (SREBP1-c) gene expression involved in lipid synthesis. So, mitochondrial up-regulated fatty acid $\beta$-oxidation activity by PPE is probably involved in the lower liver lipid accumulation with the high-fat-high-sucrose diet, while hepatic lipogenesis does not seem to be altered.

down-regulated with the HFHS diet and up-regulated with the PPE. However, the expression of nuclear respiratory factor 1 and mitochondrial transcription factor $\mathrm{A}$ was unchanged among the three experimental groups. We observed that citrate synthase, an indicator of increased mitochondrial content, even if it may reflect also high activation of the tricarboxylic acid cycle, was not modified in the HFHS rats. In HFHS dietfed rats, maximal activities of complex II and complex II + III were significantly decreased while the PPE had no effect. Moreover, the maximal activity of complex IV was not modified whatever the diet. Interestingly, complex IV activity was significantly correlated with the liver NEFA content. Moreover, complex II and complex II + III activities were negatively correlated with liver TAG content. It is possible that this reflects modification of the composition of the lipid moiety of the mitochondrial membrane in which the complexes are embedded, thus affecting their activity. The observed alteration of the maximal activity of the mitochondrial respiratory chain complexes in the liver of HFHS die-fed rats was coherent with previous studies ${ }^{(11,60-64)}$ and with the observation of steatosis, as a dysregulation of mitochondrial function might be implicated in fat accumulation in liver ${ }^{(5)}$.

\section{Conclusion}

In the present study, we confirmed that a PPE partially prevented the accumulation of TAG in liver by regulating acetyl-CoA carboxylase phosphorylation, a key enzyme in lipid metabolism, probably through the activation of hepatic sirtuin-1 deacetylase. Up-regulated fatty acid $\beta$-oxidation activity by the PPE is probably involved in the lower liver lipid accumulation with the HFHS diet, while hepatic lipogenesis did not seem altered. Desaturase activity indexes were not modified and the PPE did not modulate liver fatty acid composition, while liver fatty acid composition mostly reflected fatty acids in dietary lipids. The PPE modulated
$P G C-1 \alpha$ expression but did not modify mitochondriogenesis and mitochondrial activity. Whether this PPE should be effective at ameliorating hepatic lipid accumulation in humans deserves further study. A diagram of the pathways affected by the PPE is provided in Fig. 3.

\section{Acknowledgements}

The authors thank L. Lepourry and B. Bonnafos for the use of the animal facilities. The authors acknowledge the Société Française de Distellerie (Mr D. Ageron), which supplied the powdered PPE.

This research received no specific grant from any funding agency in the public, commercial or not-for-profit sectors.

C. C. and C. F.-C. designed the research; M. A., F. M., G. F., M. J., J. R. and C. F.-C. conducted the research. F. M., F. C., J.-P. C., C. W.-C., C. C., M.-A. C. and C. F.-C. analysed the data; C. F.-C. wrote the paper and had responsibility for the final content. All authors read and approved the final manuscript.

The authors declare no conflict of interest.

\section{References}

1. Barness LA, Opitz JM \& Gilbert-Barness E (2007) Obesity: genetic, molecular, and environmental aspects. Am J Med Genet A 143, 3016-3034.

2. Fromenty B, Robin MA, Igoudjil A, et al. (2004) The ins and outs of mitochondrial dysfunction in NASH. Diabetes Metab 30, $121-138$.

3. Erickson SK (2009) Nonalcoholic fatty liver disease. J Lipid Res 50, Suppl., S412-S416.

4. Cave M, Deaciuc I, Mendez C, et al. (2007) Nonalcoholic fatty liver disease: predisposing factors and the role of nutrition. J Nutr Biochem 18, 184-195.

5. Mantena SK, King AL, Andringa KK, et al. (2008) Mitochondrial dysfunction and oxidative stress in the pathogenesis of alcohol- and obesity-induced fatty liver diseases. Free Radic Biol Med 44, 1259-1272.

6. Baur JA, Pearson KJ, Price NL, et al. (2006) Resveratrol improves health and survival of mice on a high-calorie diet. Nature $\mathbf{4 4 4}$, 337-342.

7. Mulvihill EE, Allister EM, Sutherland BG, et al. (2009) Naringenin prevents dyslipidemia, apoB overproduction and hyperinsulinemia in LDL-receptor null mice with diet-induced insulin resistance. Diabetes 58, 2198-2210.

8. Yalniz M, Bahcecioglu IH, Kuzu N, et al. (2007) Preventive role of genistein in an experimental non-alcoholic steatohepatitis model. J Gastroenterol Hepatol 22, 2009-2014.

9. Kuzu N, Bahcecioglu IH, Dagli AF, et al. (2008) Epigal locatechin gallate attenuates experimental non-alcoholic steatohepatitis induced by high fat diet. J Gastroenterol Hepatol 23, e465-e 470 .

10. Shang J, Chen LL, Xiao FX, et al. (2008) Resveratrol improves non-alcoholic fatty liver disease by activating AMP-activated protein kinase. Acta Pharmacol Sin 29, 698-706.

11. Feillet-Coudray C, Sutra T, Fouret G, et al. (2009) Oxidative stress in rats fed a high-fat high-sucrose diet and preventive effect of polyphenols: involvement of mitochondrial and $\mathrm{NAD}(\mathrm{P}) \mathrm{H}$ oxidase systems. Free Radic Biol Med 46, 624-632.

12. Ajmo JM, Liang X, Rogers CQ, et al. (2008) Resveratrol alleviates alcoholic fatty liver in mice. Am J Physiol Gastrointest Liver Physiol 295, G833-G842. 
13. Klaus S, Pultz S, Thone-Reineke C, et al. (2005) Epigallocatechin gallate attenuates diet-induced obesity in mice by decreasing energy absorption and increasing fat oxidation. Int $J$ Obes (Lond) 29, 615-623.

14. Ogino Y, Osada K, Nakamura S, et al. (2007) Absorption of dietary cholesterol oxidation products and their downstream metabolic effects are reduced by dietary apple polyphenols. Lipids 42, 151-161.

15. Zang M, Xu S, Maitland-Toolan KA, et al. (2006) Polyphenols stimulate AMP-activated protein kinase, lower lipids, and inhibit accelerated atherosclerosis in diabetic LDL receptor-deficient mice. Diabetes 55, 2180-2191.

16. Hou X, Xu S, Maitland-Toolan KA, et al. (2008) SIRT1 regulates hepatocyte lipid metabolism through activating AMP-activated protein kinase. J Biol Chem 283, 20015-20026.

17. Csiszar A, Labinskyy N, Pinto JT, et al. (2009) Resveratrol induces mitochondrial biogenesis in endothelial cells. Am J Physiol Heart Circ Physiol 297, H13-H20.

18. Nanji AA (2004) Animal models of nonalcoholic fatty liver disease and steatohepatitis. Clin Liver Dis 8, 559-574, ix.

19. Ahmed U, Redgrave TG \& Oates PS (2009) Effect of dietary fat to produce non-alcoholic fatty liver in the rat. $J$ Gastroenterol Hepatol 24, 1463-1471.

20. Reeves PG, Nielsen FH \& Fahey GC Jr (1993) AIN-93 purified diets for laboratory rodents: final report of the American Institute of Nutrition ad hoc writing committee on the reformulation of the AIN-76A rodent diet. J Nutr 123, 1939-1951.

21. Bradford MM (1976) A rapid and sensitive method for the quantitation of microgram quantities of protein utilizing the principle of protein-dye binding. Anal Biochem 72, 248-254.

22. Folch J, Lees M \& Sloane Stanley GH (1957) A simple method for the isolation and purification of total lipides from animal tissues. J Biol Chem 226, 497-509.

23. Bartlett GR (1959) Phosphorus assay in column chromatography. J Biol Chem 234, 466-468.

24. Lepage G \& Roy CC (1986) Direct transesterification of all classes of lipids in a one-step reaction. J Lipid Res 27, 114-120.

25. Biggemann B, Laryea MD, Schuster A, et al. (1988) Status of plasma and erythrocyte fatty acids and vitamin A and E in young children with cystic fibrosis. Scand J Gastroenterol Suppl 143, 135-141.

26. Stefan N, Peter A, Cegan A, et al. (2008) Low hepatic stearoylCoA desaturase 1 activity is associated with fatty liver and insulin resistance in obese humans. Diabetologia 51, 648-656.

27. Rustin P, Chretien D, Bourgeron T, et al. (1994) Biochemical and molecular investigations in respiratory chain deficiencies. Clin Chim Acta 228, 35-51.

28. Wharton D \& Tzagoloff A (1967) Cytochrome oxidase from beef heart mitochondria. Methods Enzymol 10, 245-250.

29. Srere P (1969) Citrate synthase. Methods Enzymol 13, 3-11.

30. Scalbert A \& Williamson G (2000) Dietary intake and bioavailability of polyphenols. J Nutr 130, Suppl. 8S, 2073S-2085S.

31. Yang R \& Barouch LA (2007) Leptin signaling and obesity: cardiovascular consequences. Circ Res 101, 545-559.

32. Bargalló MV, Grau AA, Fernández-Larrea J, et al. (2006) Moderate red-wine consumption partially prevents body weight gain in rats fed a hyperlipidic diet. J Nutr Biochem 17, 139-142.

33. Decorde K, Teissedre PL, Sutra T, et al. (2009) Chardonnay grape seed procyanidin extract supplementation prevents high-fat dietinduced obesity in hamsters by improving adipokine imbalance and oxidative stress markers. Mol Nutr Food Res 53, 659-666.

34. Zheng G, Sayama K, Okubo T, et al. (2004) Anti-obesity effects of three major components of green tea, catechins, caffeine and theanine, in mice. In Vivo 18, 55-62.

35. Tessari P, Coracina A, Cosma A, et al. (2009) Hepatic lipid metabolism and non-alcoholic fatty liver disease. Nutr Metab Cardiovasc Dis 19, 291-302.
36. Postic C \& Girard J (2008) Contribution of de novo fatty acid synthesis to hepatic steatosis and insulin resistance: lessons from genetically engineered mice. J Clin Invest 118, 829-838.

37. Kim WS, Lee YS, Cha SH, et al. (2009) Berberine improves lipid dysregulation in obesity by controlling central and peripheral AMPK activity. Am J Physiol Endocrinol Metab 296, E812-E819.

38. Jang EM, Choi MS, Jung UJ, et al. (2008) Beneficial effects of curcumin on hyperlipidemia and insulin resistance in highfat-fed hamsters. Metabolism 57, 1576-1583.

39. Wang JQ, Li J, Zou YH, et al. (2009) Preventive effects of total flavonoids of Litsea coreana leve on hepatic steatosis in rats fed with high fat diet. J Ethnopharmacol 121, 54-60.

40. Quesada H, Del Bas JM, Pajuelo D, et al. (2009) Grape seed proanthocyanidins correct dyslipidemia associated with a highfat diet in rats and repress genes controlling lipogenesis and VLDL assembling in liver. Int J Obes (Lond) 33, 1007-1012.

41. Sealls W, Gonzalez M, Brosnan MJ, et al. (2008) Dietary polyunsaturated fatty acids (C18:2 $\omega 6$ and C18:3 $\omega 3)$ do not suppress hepatic lipogenesis. Biochim Biophys Acta 1781, 406-414.

42. Morise A, Mourot J, Boue C, et al. (2006) Gender-related response of lipid metabolism to dietary fatty acids in the hamster. Br J Nutr 95, 709-720.

43. Li ZZ, Berk M, McIntyre TM, et al. (2009) Hepatic lipid partitioning and liver damage in nonalcoholic fatty liver disease: role of stearoyl-CoA desaturase. J Biol Chem 284, 5637-5644.

44. Howitz KT, Bitterman KJ, Cohen HY, et al. (2003) Small molecule activators of sirtuins extend Saccharomyces cerevisiae lifespan. Nature 425, 191-196.

45. Lagouge M, Argmann C, Gerhart-Hines Z, et al. (2006) Resveratrol improves mitochondrial function and protects against metabolic disease by activating SIRT1 and PGC-1 $\alpha$. Cell 127, 1109-1122.

46. Rodgers JT, Lerin C, Haas W, et al. (2005) Nutrient control of glucose homeostasis through a complex of PGC- $1 \alpha$ and SIRT1. Nature 434, 113-118.

47. Munday MR, Milic MR, Takhar S, et al. (1991) The short-term regulation of hepatic acetyl-CoA carboxylase during starvation and re-feeding in the rat. Biochem $J$ 280, 733-737.

48. Hastings IM \& Hill WG (1990) Analysis of lines of mice selected for fat content. 2. Correlated responses in the activities of enzymes involved in lipogenesis. Genet Res 55, 55-61.

49. Lin CL, Huang HC \& Lin JK (2007) Theaflavins attenuate hepatic lipid accumulation through activating AMPK in human HepG2 cells. J Lipid Res 48, 2334-2343.

50. Begriche K, Igoudjil A, Pessayre D, et al. (2006) Mitochondrial dysfunction in NASH: causes, consequences and possible means to prevent it. Mitochondrion 6, 1-28.

51. Hong YH, Varanasi US, Yang W, et al. (2003) AMP-activated protein kinase regulates $\mathrm{HNF} 4 \alpha$ transcriptional activity by inhibiting dimer formation and decreasing protein stability. $J$ Biol Chem 278, 27495-27501.

52. Hayhurst GP, Lee YH, Lambert G, et al. (2001) Hepatocyte nuclear factor $4 \alpha$ (nuclear receptor $2 \mathrm{~A} 1$ ) is essential for maintenance of hepatic gene expression and lipid homeostasis. Mol Cell Biol 21, 1393-1403.

53. Wakil SJ, Stoops JK \& Joshi VC (1983) Fatty acid synthesis and its regulation. Аппи Rev Biochem 52, 537-579.

54. Lin J, Handschin C \& Spiegelman BM (2005) Metabolic control through the PGC-1 family of transcription coactivators. Cell Metab 1, 361-370.

55. Medina-Gomez G, Gray S \& Vidal-Puig A (2007) Adipogenesis and lipotoxicity: role of peroxisome proliferator-activated receptor $\gamma($ PPAR $\gamma)$ and PPAR $\gamma$ coactivator-1 (PGC1). Public Health Nutr 10, 1132-1137.

56. Fromenty B \& Pessayre D (1995) Inhibition of mitochondrial $\beta$-oxidation as a mechanism of hepatotoxicity. Pharmacol Ther 67, 101-154. 
57. Raederstorff DG, Schlachter MF, Elste V, et al. (2003) Effect of EGCG on lipid absorption and plasma lipid levels in rats. J Nutr Biochem 14, 326-332.

58. Koo SI \& Noh SK (2007) Green tea as inhibitor of the intestinal absorption of lipids: potential mechanism for its lipid-lowering effect. J Nutr Biochem 18, 179-183.

59. Wallace DC (2005) A mitochondrial paradigm of metabolic and degenerative diseases, aging, and cancer: a dawn for evolutionary medicine. Апnи Rev Genet 39, 359-407.

60. Iossa S, Lionetti L, Mollica MP, et al. (1999) Fat balance and hepatic mitochondrial function in response to fat feeding in mature rats. Int J Obes Relat Metab Disord 23, 1122-1128.
61. Iossa S, Lionetti L, Mollica MP, et al. (2000) Effect of longterm high-fat feeding on energy balance and liver oxidative activity in rats. Br J Nutr 84, 377-385.

62. Iossa S, Lionetti L, Mollica MP, et al. (2003) Effect of high-fat feeding on metabolic efficiency and mitochondrial oxidative capacity in adult rats. Br J Nutr 90, 953-960.

63. Perez-Carreras M, Del Hoyo P, Martin MA, et al. (2003) Defective hepatic mitochondrial respiratory chain in patients with nonalcoholic steatohepatitis. Hepatology 38, 999-1007.

64. Garcia-Ruiz I, Rodriguez-Juan C, Diaz-Sanjuan T, et al. (2006) Uric acid and anti-TNF antibody improve mitochondrial dysfunction in ob/ob mice. Hepatology 44, 581-591. 